\title{
LONG-TERM DYNAMICS AND THE ORBITAL INCLINATIONS OF THE CLASSICAL KUIPER BELT OBJECTS
}

\author{
MARC J. KUCHNER ${ }^{1}$ \\ Harvard-Smithsonian Center for Astrophysics, Mail Stop 20,60 Garden Street, Cambridge, MA 02138; mkuchner@cfa.harvard.edu \\ Michael E. Brown \\ Division of Planetary Sciences, California Institute of Technology, Pasadena, CA 91125; mbrown@caltech.edu \\ AND \\ Matthew Holman \\ Harvard-Smithsonian Center for Astrophysics, Mail Stop 18,60 Garden Street, Cambridge, MA 02138; mholman@cfa.harvard.edu \\ Received 2002 January 7; accepted 2002 May 3
}

\begin{abstract}
We numerically integrated the orbits of 1458 particles in the region of the classical Kuiper belt (41 AU $\leq a \leq 47 \mathrm{AU}$ ) to explore the role of dynamical instabilities in sculpting the inclination distribution of the classical Kuiper belt objects (KBOs). We find that the selective removal of low-inclination objects by overlapping secular resonances $\left(\nu_{17}\right.$ and $\left.\nu_{18}\right)$ acts to raise the mean inclination of the surviving population of particles over 4 billion yr of interactions with Jupiter, Saturn, Uranus, and Neptune, though these long-term dynamical effects do not themselves appear to explain the discovery of KBOs with inclinations near $30^{\circ}$. Our integrations also imply that after 3 billion yr of interaction with the massive planets, high-inclination KBOs more efficiently supply Neptune-encountering objects, the likely progenitors of short-period comets, Centaurs, and scattered KBOs. The secular resonances at low inclinations may indirectly cause this effect by weeding out objects unprotected by mean motion resonances during the first 3 billion yr.
\end{abstract}

Key words: celestial mechanics — comets: general — Kuiper belt — methods: $N$-body simulations planetary systems: formation

\section{INTRODUCTION}

Most of the mass of the Kuiper belt between 30 and 50 AU appears to reside in a region outside semimajor axis $a=40$ AU called the classical Kuiper belt (Jewitt et al. 1998). Early Kuiper belt surveys found that Kuiper belt objects (KBOs) can have surprisingly high orbital inclinations (Jewitt \& Luu 1995; Jewitt et al. 1996). Recent largescale surveys (Jewitt et al. 1998; Trujillo et al. 2001) have established that the classical KBOs-not just the relatively nearby Plutinos - frequently have high orbital inclinations $\left(i \gtrsim 15^{\circ}\right)$.

The discovery of these high-inclination objects seems to point to unknown processes in the primordial solar system. Several mechanisms for "pumping up" the ancient KBO inclination distribution have been investigated, such as resonant encounters (Malhotra 1995; Nagasawa \& Ida 2000) and perturbations from passing stars (Ida et al. 2000), scattered planets (Thommes et al. 2002), and planetesimals (Petit et al. 1999). Large KBOs that may have existed long ago during the epoch of planet formation could also have stirred the KBO orbits (Kenyon 2002).

However, theories of the ancient solar system alone cannot explain the current KBO orbital distribution. Orbit integrations in the Kuiper belt (Torbett 1989; Torbett \& Smoluchowski 1990; Gladman \& Duncan 1990; Levison \& Duncan 1993; Holman \& Wisdom 1993) have shown that the dynamical effects of the massive planets on the Kuiper belt have probably removed most of the original KBOs over the lifetime of the solar system. Objects removed recently by these processes probably supply today's population of Cen-

\footnotetext{
${ }^{1}$ Michelson Postdoctoral Fellow.
}

taurs, short-period comets, and scattered KBOs (Fernández 1980; Duncan et al. 1988). One might ask whether selective removal of objects by interactions with the planets has altered the inclination distribution of the surviving population.

Furthermore, debiased estimates of the $\mathrm{KBO}$ inclination distribution (Brown 2001) suggest that the classical KBOs divide into two populations: one dynamically warm, with a typical inclination of $i \approx 17^{\circ}$, and one dynamically cold, with a typical inclination $i \approx 2^{\circ}$. We wonder whether longterm dynamical interactions helped create the apparent two-component distribution.

To understand the role of dynamical stability in shaping the classical KBO inclination distribution and creating Centaurs, comets, and scattered KBOs, we performed a new large-scale simulation of the dynamics of KBOs, integrating the orbits of 1458 test particles in the region of the classical Kuiper belt under the influence of gravity from Jupiter, Saturn, Uranus, and Neptune. Duncan et al. (1995) performed the most recent previous large-scale integration of orbits in the Kuiper belt. They used an initial grid with fine resolution in semimajor axis, but they only sampled a few initial inclinations and did not integrate the orbits of high-inclination objects $i>1^{\circ}$ for longer than 1 billion yr. Our work complements theirs; at the cost of coverage in semimajor axis we focus on exploring the consequences of high inclination and eccentricity over a 4 billion yr period.

\section{THE OBSERVED CLASSICAL KBO ORBITS}

Figure 1 shows the orbital eccentricities and inclinations with respect to the ecliptic plane of the 410 known classical KBOs with 40.5 AU $<a<47.5 \mathrm{AU}$ sorted into seven pan- 


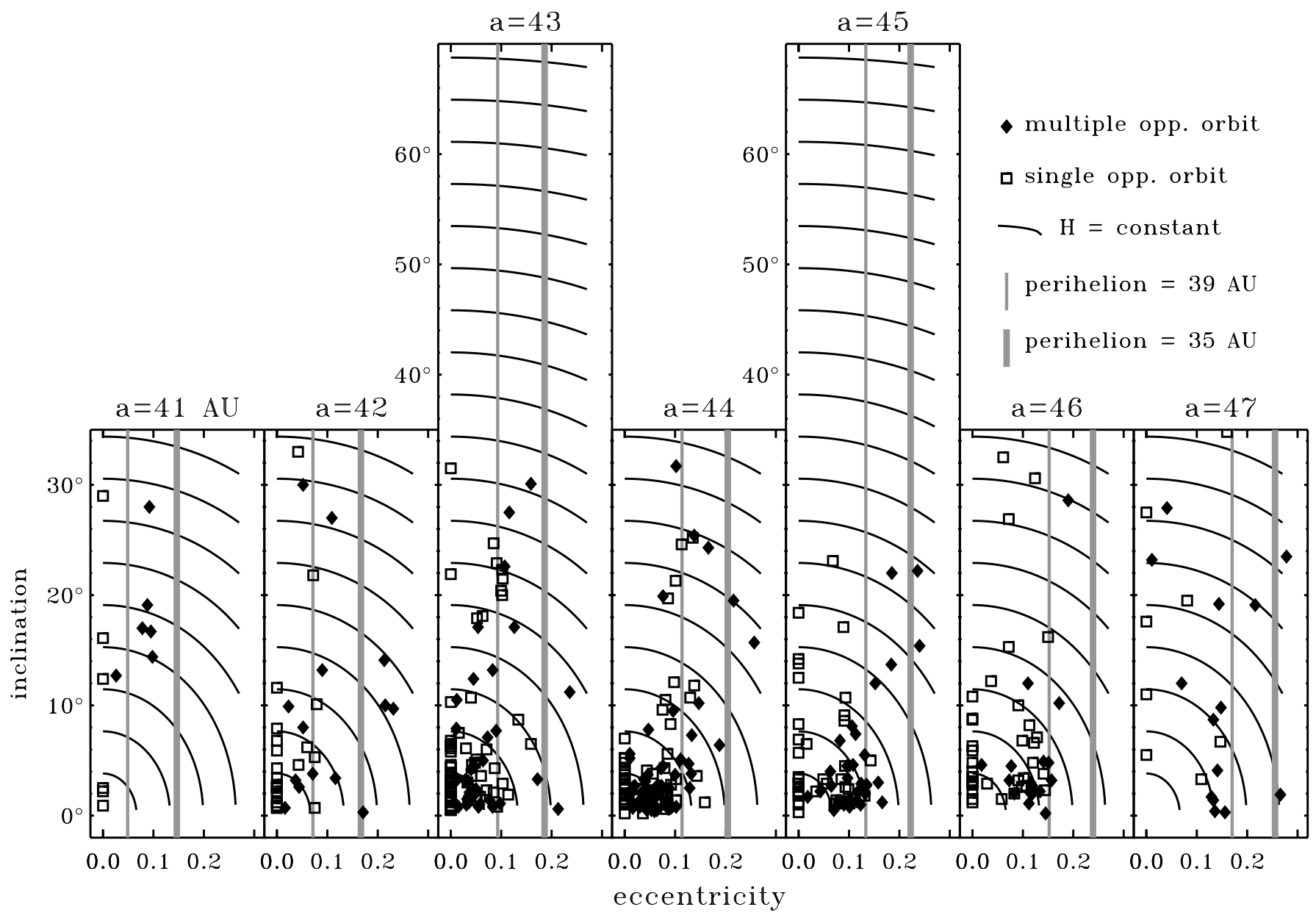

FIG. 1.-Inclinations and eccentricities of the known classical KBOs sorted by semimajor axis rounded to the nearest AU. The vertical lines indicate perihelion distances of 35 and $39 \mathrm{AU}$, and the curves show constant values of $H=\left[G M_{\odot} a\left(1-e^{2}\right)\right]^{1 / 2} \cos i$, the component of angular momentum perpendicular to the ecliptic plane.

els by semimajor axis $a$, rounded to the nearest AU. We obtained these data from the Minor Planet Center's Web site. ${ }^{2}$ Filled symbols indicate orbits for KBOs observed at multiple oppositions. The single-opposition orbits, shown as empty squares, generally have accurate inclinations but poorly measured eccentricities; often the Minor Planet Center assumes zero eccentricity for single-opposition orbits.

These classical KBOs have a mean inclination of 6.6 . However, the data reflect a strong observational bias against detecting high-inclination KBOs; high-inclination objects spend less time near the ecliptic plane than low-inclination objects. The figure also reflects severe observational biases against detecting distant KBOs. The brightness of a $\mathrm{KBO}$ in reflected light decreases roughly as $1 / r^{4}$, where $r$ is the KBO's heliocentric distance. This distance bias propagates into correlated biases in semimajor axis, eccentricity, and mean anomaly since most KBOs are discovered near perihelion. See Trujillo \& Brown (2001) and Gladman et al. (2001) for recent discussions of the radial distribution of KBOs.

Plotted on Figure 1 is a family of curves corresponding to constant values of $H=\left[G M_{\odot} a\left(1-e^{2}\right)\right]^{1 / 2} \cos i$, the com-

${ }^{2}$ At http://cfa-www.harvard.edu/iau/mpc.html. ponent of the orbital angular momentum (per particle mass) in the direction perpendicular to the ecliptic plane. $H$ is largest in the lower left-hand corner of each plot $(i=e=0)$. In a system where all the planets occupy circular orbits in the same plane, the orbit of a test particle conserves the component of its angular momentum perpendicular to that plane (Kozai 1962; Thomas \& Morbidelli 1996). We would expect the test particles in our simulation to conserve $H$ and only evolve parallel to these curves in the absence of semimajor axis variations if all the perturbing planets had $i=e=0$ with respect to the ecliptic plane.

Figure 2 shows the eccentricities and semimajor axes of the known KBOs observed at more than one opposition. Two curves on this figure show the eccentricities corresponding to perihelion distances of $q=30.1 \mathrm{AU}$ (the Neptune crossing line) and $q=39$ AU. Also, each panel in Figure 1 has vertical gray lines indicating $q=35 \mathrm{AU}$ and $q=39$ AU. The dearth of objects with $q$ much greater than 39 AU in Figure 2 shows the bias toward detecting objects with small perihelia.

Forty-seven of the classical KBOs with observations at multiple oppositions have orbits with perihelia less than 39 AU. These 47 objects have a mean inclination of $13^{\circ}$, much higher than the mean inclination of the classical KBOs as a whole (6.6). Only 11 of these 47 have orbits with perihelion 


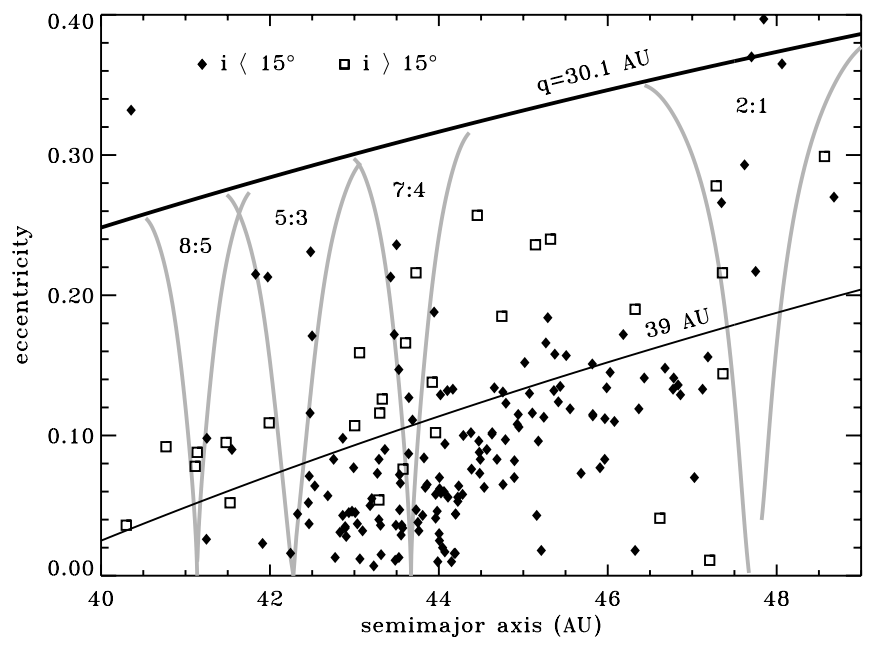

FIG. 2.-Eccentricity vs. semimajor axis for the orbits of the known classical KBOs observed at more than one opposition. High-inclination orbits $\left(i>15^{\circ}\right)$ are squares; low-inclination $\left(i<15^{\circ}\right)$ orbits are filled diamonds. Gray curves indicate numerically computed boundaries of mean motion resonances (MMRs) with Neptune, and black curves show where the perihelion distance $q$ is $30.1 \mathrm{AU}$; i.e., the KBOs become Neptune crossing (thick curve) and where $q=39 \mathrm{AU}$ (thin curve).

$q<35$ AU. Duncan et al. (1995) found that particles with $q<35$ AU generally did not survive 1 billion yr of interaction with the giant planets.

Gray curves in Figure 2 indicate the boundaries of the $8: 5,5: 3,7: 4$, and $2: 1$ mean motion resonances (MMRs) with Neptune, using the planar circular restricted threebody problem as a model for the motion of small bodies in the field of the Sun and Neptune. We numerically computed these boundaries following the technique developed by Ferraz-Mello \& Sato (1989) to avoid relying on analytic expansions of the disturbing function which invoke low eccentricities. Morbidelli et al. (1995) first applied this approach to MMRs in the Kuiper belt. The resonance widths calculated this way contain a chaotic zone along the boundary at high eccentricities, while the widths calculated by Malhotra (1996) exclude this region. This chaos may affect the long-term dynamics and stability of KBOs along the resonance boundaries.

\section{ORBIT INTEGRATIONS}

We integrated the orbits of 1458 noninteracting particles for 4 billion yr under the gravitational influences of Jupiter, Saturn, Uranus, Neptune, and the Sun using the MVS symplectic integrator from the SWIFT package by Levison \& Duncan (1994) based on an algorithm by Wisdom \& Holman (1991). The initial arguments of pericenter, mean longitudes, and ascending nodes were chosen randomly from a uniform distribution on the interval $[0,2 \pi)$. We used orbital elements referred to the ecliptic plane, rather than the invariable plane, so they can easily be compared with observations. We used orbital data for the planets from Cohen et al. (1973) and time steps of $1 \mathrm{yr}$. The integration required donated time on 20 different Sun workstations at the Caltech Department of Astronomy and at the HarvardSmithsonian Center for Astrophysics. To minimize network traffic, we recorded the orbital elements of each particle only once every 10 million yr.
To check that our choice of a 1 yr step size was small enough, we integrated the orbits of 100 objects for a billion years in the vicinity of a subtle high-inclination feature $(a=42, e=0, i=[60,60.02,60.04, \ldots, 61.98]$; see $\S 4.2)$, using a step size of $1 \mathrm{yr}$. Then we repeated the integration using the same initial conditions and a step size of one-quarter of a year. Naturally, the final orbits differed as a result of the nonlinearity of the dynamics and the long integration times. But the final distributions of the orbits in eccentricity and inclination space were indistinguishable.

Our initial population of objects had semimajor axes of $\{41,42,43, \ldots, 47\} \mathrm{AU}$, eccentricities of $\{0,1 / 30$, $2 / 30, \ldots, 8 / 30\}$, and inclinations of $\{0,1 / 30,2 / 30, \ldots$, $17 / 30\} \mathrm{rad}\left(0.0000,1.9099,3.8197, \ldots, 32^{\circ} 4676\right)$. We refer to this grid of 1134 particles, which evenly fills a rectangular prism in $(a, e, i)$ space, as our first batch. We also performed some exploratory integrations with higher initial inclinations; at 43 and $45 \mathrm{AU}$, we started particles with inclinations up to $1.1666 \mathrm{rad}\left(66^{\circ} 8451\right)$. We removed particles from the integration when they encountered Neptune's Hill sphere. Six hundred and twenty-six particles from the first batch survived the entire integration.

\subsection{Semimajor Axis Evolution}

Though the particles began with integral values of semimajor axis, after 10 million yr of integration only a shadow of the discrete initial conditions remained. If we combine all the data in the first batch, we find that by $t=10$ million yr the particles have spread out in semimajor axis into a distribution with half-width at half maximum of $0.4 \mathrm{AU}$. At $t=4$ billion yr the core of the distribution has not changed, but the particles in the wings are gone. Only $0.1 \%$ of the particles that survived for 4 billion yr were transported farther than $0.7 \mathrm{AU}$ from their initial semimajor axes. We infer that the observed classical KBOs have not strayed far in semimajor axis since the planets settled into their current configuration-unless another effect besides long-term interactions with the planets disturbed their orbits.

Figure 3 shows the eccentricities and semimajor axes of the particles that survived for 4 billion yr. The eccentricities

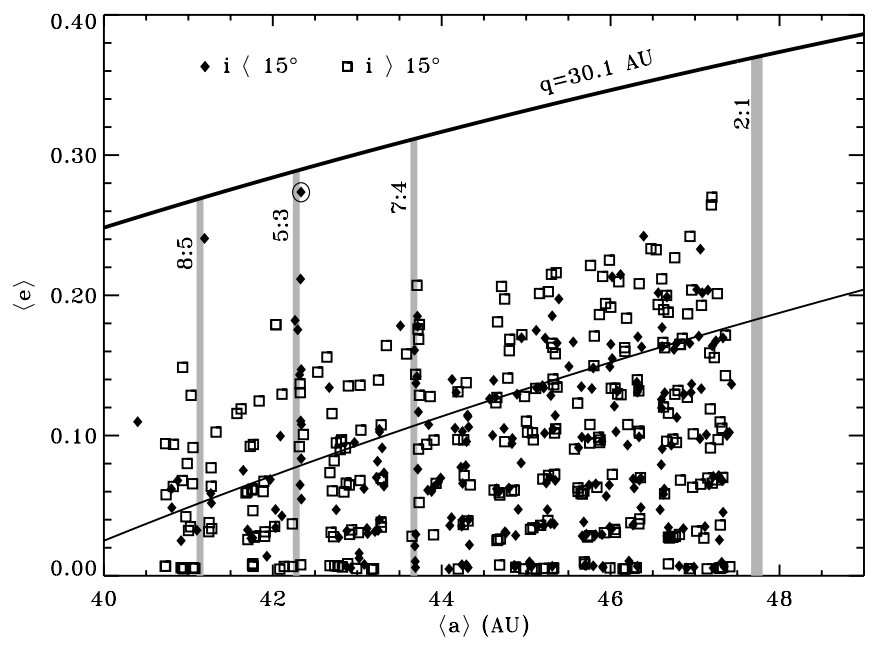

FIG. 3.-Mean eccentricity vs. mean semimajor axis for the particles in the first batch of our integration that survived for 4 billion yr, averaged over the period $t=3$ billion to $t=4$ billion yr. Vertical gray lines indicate the nominal locations of MMRs with Neptune, and black curves show where $q=30.1 \mathrm{AU}$ (thick curve) and where $q=39 \mathrm{AU}$ (thin curve). 


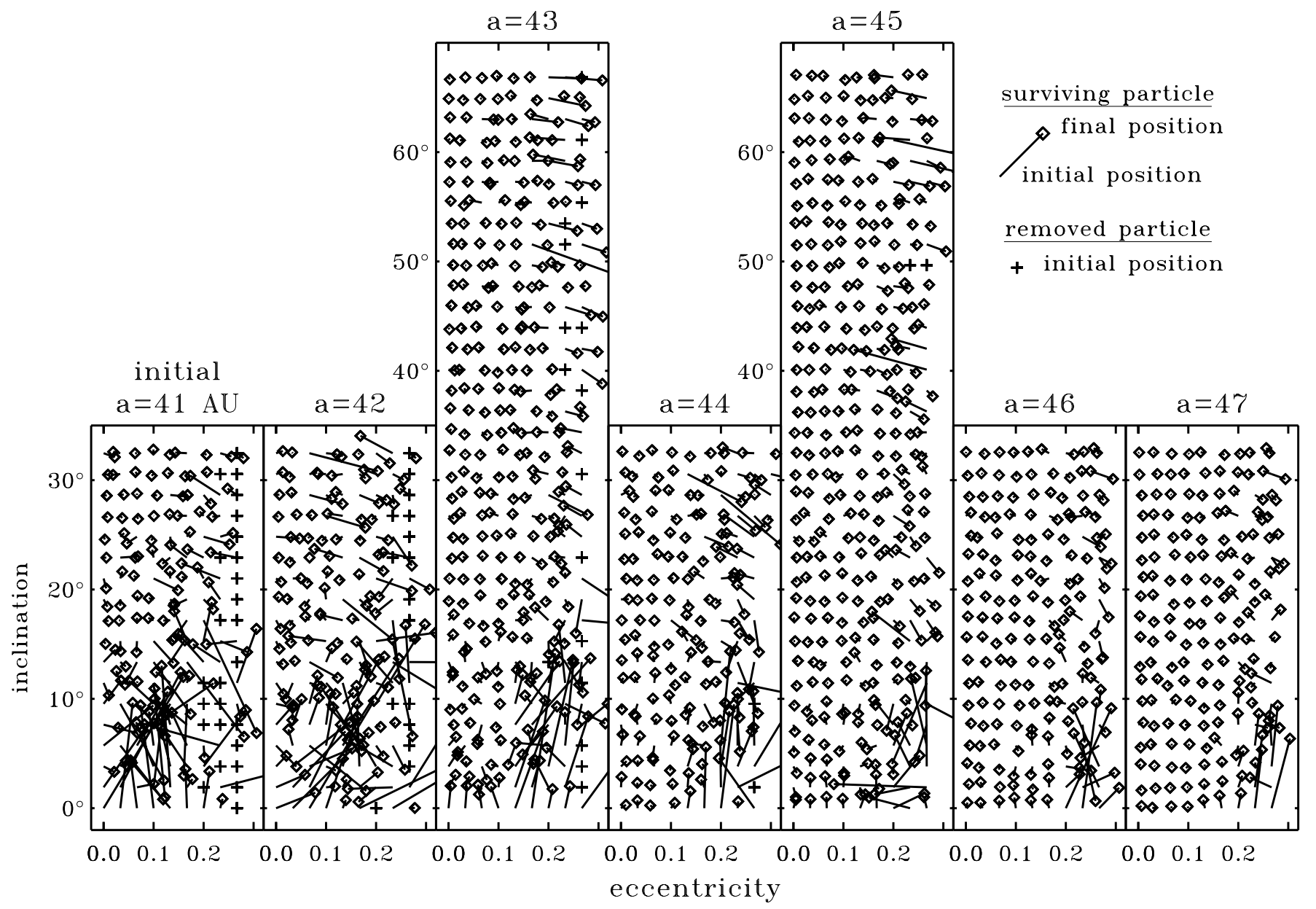

FIG. 4.-After 10 million yr the orbits of our test particles in the classical Kuiper belt have evolved to the locations indicated by the diamonds. Short lines connect each diamond to the initial orbit. Crosses show the initial locations of particles that have been removed. The particles are sorted into seven panels by their initial semimajor axes.

and semimajor axes have been averaged over the period 3-4 billion yr (100 data dumps). Solid diamonds show the averaged orbits of low-inclination test particles $\left(i<15^{\circ}\right)$; empty squares show the averaged orbits of high-inclination particles $\left(15^{\circ} \leq i<30^{\circ}\right)$. In the lower right particles at low $e$ and high $a$ remain clustered, reflecting the initial conditions. This zone appears to be stable for the lifetime of the solar system; however, few KBOs are known with high- $a$ and low-e orbits since the perihelia of these orbits are far from the Sun. To the left of the figure, at $a<44$ AU, an inclination-dependent instability appears to have removed many of the low-inclination particles, particularly at perihelia $q<39$ AU.

The gray vertical lines indicate the nominal positions of the $8: 5,5: 3,7: 4$, and $2: 1$ MMRs. Since the plotted orbits are averaged over the resonant librations, the libration widths of the resonances do not appear. Figures 2 and 3 show that objects with small perihelion distances $q$ often inhabit MMRs, which can protect them from close encounters with Neptune. The MMRs at $a<44$ AU appear to serve double protective duty; they protect low- $q$ objects from close encounters, and they protect low-inclination objects from ejection by the instability that removes lowinclination particles (see $\S \S 4.1$ and 6).

\subsection{Eccentricity and Inclination Evolution}

Figure 4 shows the orbits of the test particles after 10 million yr of integration. The particles are sorted into seven panels by their initial semimajor axes. Diamonds indicate the orbital eccentricity and inclination of the particles that survived the integration for 10 million yr. Thin lines connect the diamonds to the initial orbits. Crosses indicate the initial positions of particles that suffered close encounters with Neptune.

Even after this relatively short period many of the particles at low inclinations, $i<15^{\circ}$, have been substantially perturbed, their inclinations raised. Particles with inclinations $i \gtrsim 20^{\circ}$ have generally evolved along lines of $H=$ constant, shown in Figure 1. But so far only a few lowinclination particles have been removed.

Figure 5 shows the state of affairs after 4 billion yr, summarizing the inclination and eccentricity evolution of the particles in the same manner as Figure 4. This figure should be compared with the current distribution of $\mathrm{KBO}$ orbits (Fig. 1). By this time most of the low-inclination particles whose orbits were greatly perturbed (the diamonds with the long lines in Fig. 4) have now been ejected. By 4 billion yr nearly all of the objects with initial perihelion $q<35 \mathrm{AU}$ 


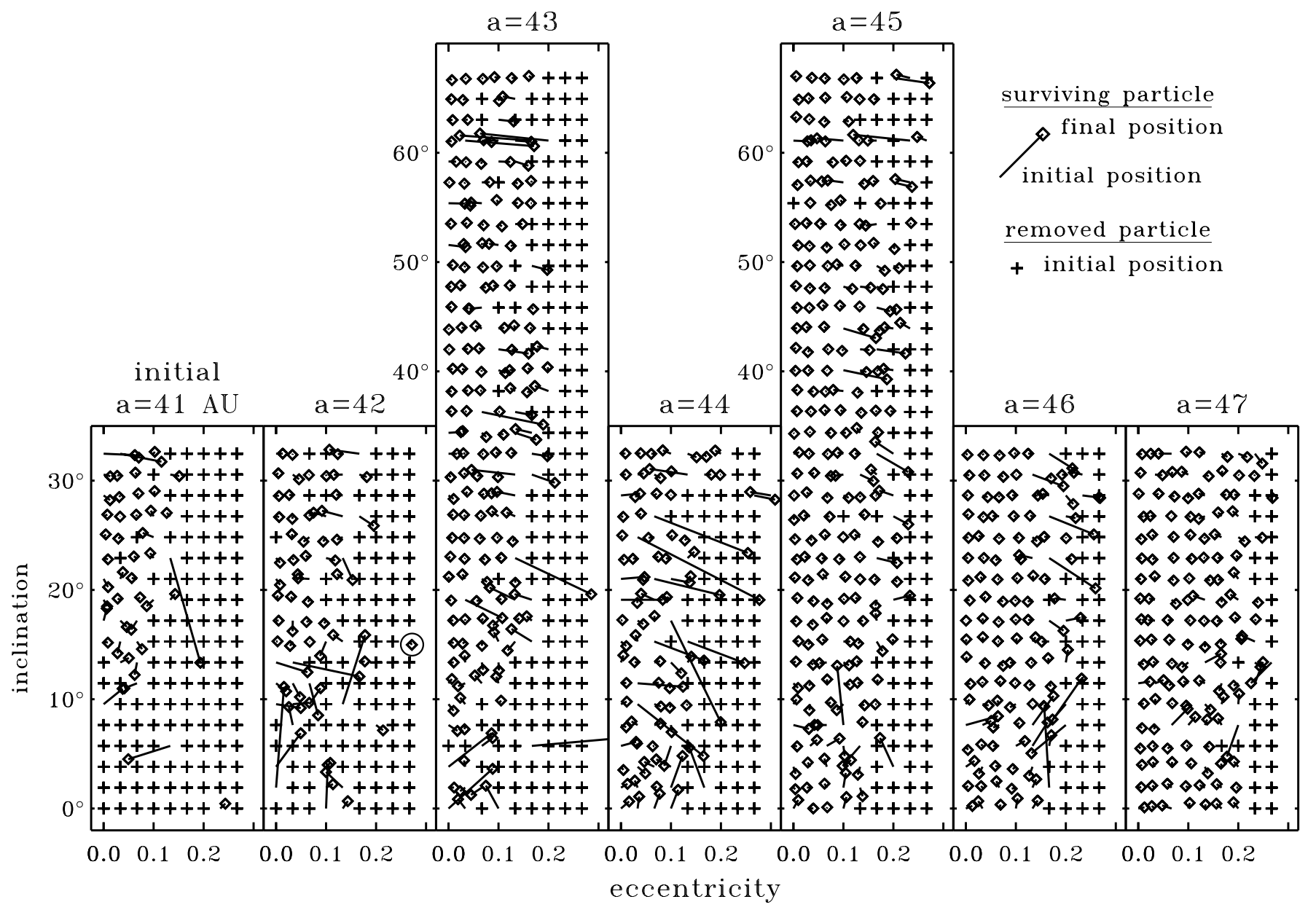

FIG. 5.-After four billion yr, most of the test particles in the classical Kuiper belt whose orbits had dramatically evolved in the first 10 million yr are gone. The $\nu_{8}, \nu_{18}$, and $\nu_{17}$ secular resonances have removed some low-inclination particles at initial semimajor axes $a=41$ and $42 \mathrm{AU}$. Evolution at high inclinations is mostly parallel to the curves of $H=$ constant shown in Fig. 1.

have been removed. This cutoff is not even: several objects have been transported into the region $q<35 \mathrm{AU}$, and at low inclinations, $i \lesssim 10^{\circ}$, the border of the unstable region is closer to $q=38 \mathrm{AU}$ (see Fig. 1). However, Figure 5 shows a systematic removal of high-eccentricity objects, which vividly demonstrates that creating the high-inclination KBOs does not require a special mechanism that only increases inclinations and not eccentricities.

When we discuss the relevance of our simulations to the $\mathrm{KBO}$ inclination distribution, we find it convenient to define two zones in the classical Kuiper belt, one at 40.5 $\mathrm{AU}<a<44 \mathrm{AU}$, the other at $44 \mathrm{AU}<a<47.5 \mathrm{AU}$. This division cuts the distribution of known KBOs roughly in half; of the classical KBOs plotted in Figure 1, 213 fall in the first group (84 with multiple opposition orbits) and 197 fall the second group (92 with multiple opposition orbits). The debiased estimate of the radial distribution of KBOs by Trujillo \& Brown (2001) is also roughly symmetric about 44 AU. As Figure 3 shows, most of the inclination-dependent structure after 4 billion yr is located in the first zone, at $a<44 \mathrm{AU}$.

Figure 6 summarizes the inclination distributions of the two zones, using the first batch of particles $\left(i<33^{\circ}\right)$ after 10 million, 1 billion, and 4 billion yr. The semimajor axis limits are applied when the distributions are evaluated. The 1 and
4 billion yr distributions were averaged over 100 million yr (10 data dumps) to cover periodic secular motions and provide better statistics.

After 10 million yr (dashed line) only $9 \%$ of the particles have been ejected, but many of the low-inclination objects have moved to higher inclination orbits. After 4 billion yr almost half of the particles $(46 \%)$ are gone, and the region $40.5 \mathrm{AU}<a<44 \mathrm{AU}$ has been hit the hardest, especially at low inclinations. An approximation to the final distribution at 4 billion yr (solid curves, Fig. 6) is

$$
\begin{aligned}
& n(i) \approx \\
& \begin{cases}0.20+0.0017\left(i-4^{\circ}\right)^{2} & 40.5 \mathrm{AU}<a<44 \mathrm{AU}, i<17.5 \\
0.51 & 40.5 \mathrm{AU}<a<44 \mathrm{AU}, i \geq 17.5 \\
0.7 & 44 \mathrm{AU}<a<47.5 \mathrm{AU}\end{cases}
\end{aligned}
$$

\section{THE CAUSES OF THE INSTABILITIES}

As far as we know, all dynamical instability is caused by the overlap of resonances. We performed some additional integrations to help understand the dynamics of the unstable regions in the classical Kuiper belt in this context. 

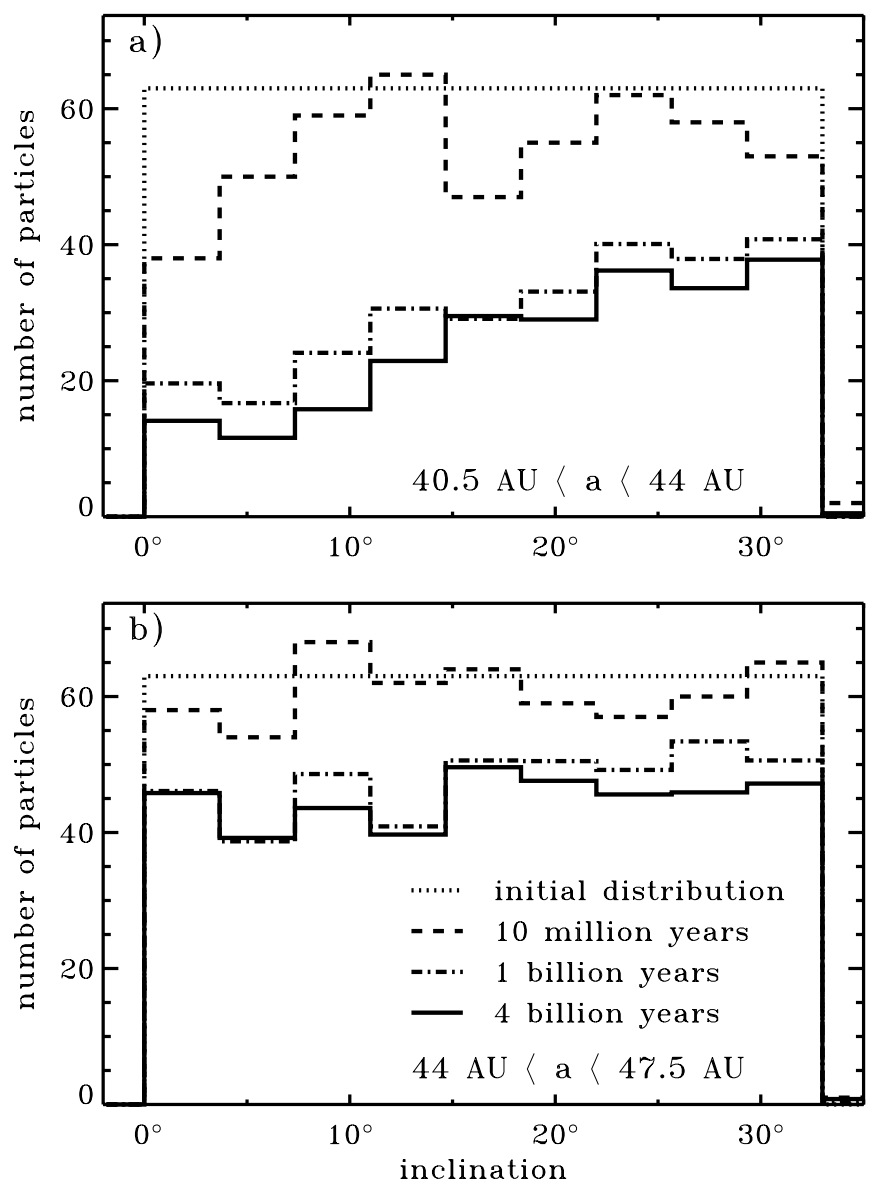

FIG. 6.-Histograms of the orbital inclinations of the surviving particles from our first batch of integrations $\left(i<33^{\circ}\right)$ after $0 \mathrm{yr}$ (dotted line), 10 million yr (dashed line), 1 billion yr (dash-dot line), and 4 billion yr (solid line). (a) Only particles with $40.5 \mathrm{AU}<a<44 \mathrm{AU}$ (b) Only particles with 44 $\mathrm{AU}<a<47.5 \mathrm{AU}$

\subsection{Secular Resonances at Low Inclinations}

The only notable secular resonances from 41-47 AU are the perihelion and node resonances with Neptune $\left(\nu_{8}\right.$ and $\left.\nu_{18}\right)$ and the node resonance with Uranus $\left(\nu_{17}\right)$, all of which are nominally located at $a<41.6$ AU (Knezevic et al. 1991). Figure 3 of Duncan et al. (1995) shows that these resonances deplete objects at low inclinations at $a=41-42$ AU. However, Figure 4 of Holman \& Wisdom (1993) shows that these resonances affect the inclinations of particles as distant as $a=50$ AU. Our Figure 4 shows in three dimensions a region of stirring in inclination and eccentricity that penetrates all of the seven panels at low inclinations and high eccentricities. This region appears to be the broad wing of the zone of influence of the overlapped secular resonances centered at 41-42 AU, shown in Figure 4 of Holman \& Wisdom (1993).

To test this identification, we integrated the orbits of 72 particles with initial $a=43 \mathrm{AU}, e=0.0, \Omega=\left\{0^{\circ}, 54^{\circ}\right.$, $\left.126^{\circ}, 198^{\circ}, 270^{\circ}, 342^{\circ}\right\}, \quad i=\{0,1 / 50,2 / 50, \ldots, 11 / 50\} \mathrm{rad}$ $\left(0^{\circ}-12.6051\right)$. Ten of these particles were removed before 4 billion yr, all from initial inclinations $\leq 6 / 50 \mathrm{rad}$ (6.875). We plotted pseudosurfaces of section for all of these particles in various coordinate systems to look for librational motion indicating the presence of a resonance.
All of the particles in this group that were ejected shared a common behavior. Figure 7 shows pseudosurfaces of section for a typical ejected particle in two coordinate systems: $\left[e \cos \left(\omega-\omega_{\mathrm{N}}\right), e \sin \left(\omega-\omega_{\mathrm{N}}\right)\right]$, and $\left[i \cos \left(\Omega-\Omega_{\mathrm{N}}\right), i \sin \right.$ $\left(\Omega-\Omega_{\mathrm{N}}\right)$ ], where $\omega$ refers to the argument of pericenter, $\Omega$ refers to the argument of ascending node, and the subscript $\mathrm{N}$ refers to Neptune. The angle $\omega-\omega_{\mathrm{N}}$ circulates, while the orbit undergoes complex motion in the inclination and $\Omega-\Omega_{\mathrm{N}}$ coordinates, alternating between libration and circulation as the inclination slowly grows. Finding this common behavior indicates that competing nodal resonances $\left(\nu_{17}\right.$ and $\left.\nu_{18}\right)$ dominate the long-term dynamics of these particles. These competing resonances appear to cause the broad swath of destabilization at low inclinations and high eccentricities apparent in Figures 3, 4, 5, and 6.

\subsection{The Kozai Resonance}

The Kozai resonance is the tendency of high-inclination orbits in the three-body problem to undergo secular libration of the argument of pericenter $\omega$, coupled with oscillations of $i$ and $e$, which conserve $H$. Recall that we measure an orbit's argument of perihelion from the ascending node, so, in the solar system, this motion corresponds to an oscillation of $\omega$ about the invariable plane. Kozai (1962) made the approximation that the planets all orbit in the same plane, and he calculated that, in the solar system, these oscillations set in at inclinations of $i>32^{\circ}$ for $a=3 \mathrm{AU}$. Thomas \& Morbidelli (1996) calculated that under the same approximation, no region of the Kuiper belt should be subject to the Kozai resonance.

But Figure 5 shows that the surviving particles around $i=61^{\circ}$ show more eccentricity evolution than other highinclination particles. The strong inclination dependence of this effect and the way the motion appears to conserve $H$ suggest that it is a Kozai phenomenon. We integrated the orbits of 24 particles with initial $a=43 \mathrm{AU}, e=\Omega=0$ at initial $\omega=\left\{0^{\circ}, 54^{\circ}, 126^{\circ}, 198^{\circ}, 270^{\circ}, 342^{\circ}\right\}$ and initial $i=\left\{60^{\circ}, 61^{\circ}, 62^{\circ}, 63^{\circ}\right\}$ and examined the evolution of their orbits to look for librations, using the coordinates $h=e \cos \omega$ and $k=e \sin \omega$. Figure 8 shows three representative pseudosurfaces of section that illustrate the three types of behavior we found in this region. Figures $8 a$ and $8 b$ show the evolution of particles that survived the integration, while Figure $8 c$ shows the evolution of one of the two particles (initial $\omega=0, i=60^{\circ}$, and $\omega=0, i=61^{\circ}$ ) that did not survive. The points in Figure $8 a$ fill a roughly circular region, indicating chaotic motion confined to low eccentricities (Henon 1983). In Figures $8 b$ and $8 c$, the motion appears to be a superposition of this chaotic motion with libration about $\omega=0^{\circ}$ or $\omega=180^{\circ}$, indicated by the loops. The librational motion indicates the influence of a Kozai resonance.

Occasionally, a particle at low inclinations in a MMR can also undergo Kozai libration (Morbidelli 1997). When we plotted pseudosurfaces of section for all of the 1458 particles in the big integration, we found one particle, with initial $a=42 \mathrm{AU}, i=15^{\circ} .278, e=0.266666$, that went into libration about $\omega=90^{\circ}$. The orbit of this particle, circled in Figures 3 and 5, is inside Neptune's 5:3 MMR. The Kozai libration may help the MMR protect the particle from doom at the hands of the overlapping secular resonances in this region of dynamical space, since the particle survives for the entire 4 billion yr of the integration. 

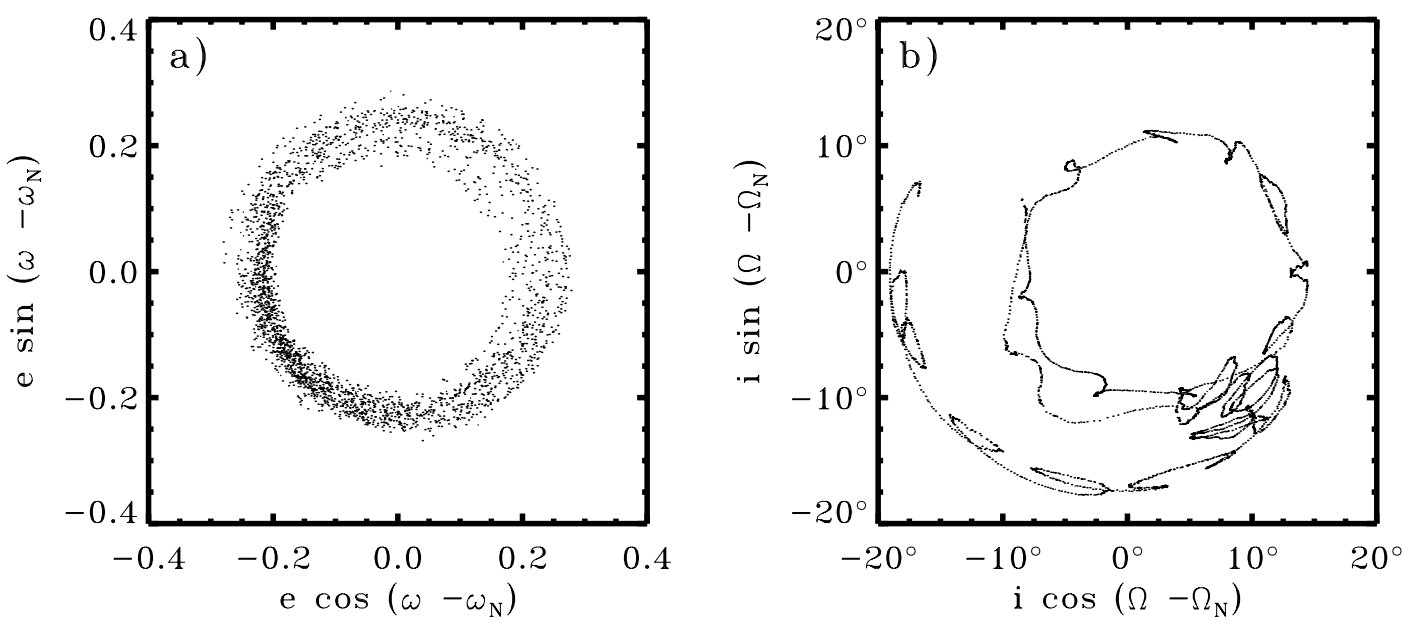

FIG. 7.-Pseudosurfaces of section for a typical ejected low-inclination particle with initial semimajor axis $a=43$ AU, eccentricity $e=0$. ( $a$ ) Polar plot of eccentricity and $\omega-\omega_{\mathrm{N}}$. (b) Polar plot of inclination and $\Omega-\Omega_{\mathrm{N}}$.

\section{THE OBSERVED KBO INCLINATION DISTRIBUTION}

As we mentioned in $\S 2$, high-inclination KBOs spend less time near the ecliptic plane than low inclination KBOs, so they evade ecliptic plane surveys. Brown (2001) realized that this observational bias could easily be corrected for the subset of low-eccentricity KBOs discovered near the ecliptic plane; for this subset, high-inclination objects will be underrepresented by the simple factor of $\sin i$, assuming no correlation between longitude and inclination. The Kozai phenomenon could produce such a correlation, but while Kozai libration may be common among Plutinos, our integrations in the classical Kuiper belt produced only one Kozai librator at inclinations $<50^{\circ}$. We used the $\sin i$ debiasing method to calculate separate debiased inclination distributions for the inner part of the classical Kuiper belt (40.5 AU $<a<44 \mathrm{AU}$ ) and the outer part of the classical Kuiper belt (44 AU $<a<47.5$ AU) considering only objects discovered at ecliptic latitude less than $1^{\circ}, 209$ objects in total. Figure 9 shows these two distributions, with error bars indicating the Poisson noise due to the limited number of objects per bin.

As Figure 6 shows, 4 billion yr of interaction with the massive planets affects the inclination distribution of particles in the outer half of the classical Kuiper belt little, but it could substantially alter the inclination distribution of particles with $a<44$ AU. Perhaps the inclination distribution of the inner half of the classical Kuiper belt (Fig. 9a) once resembled the inclination distribution of the outer half of the classical Kuiper belt (Fig. 9b). We have too few objects to make a detailed comparison, but the distribution in Figure $9 b$ clearly has relatively more low-inclination objects than the distribution in Figure $9 a$.

Figure $9 a$ shows a modest peak at $i \approx 8^{\circ}$, which piques our curiosity. After only 10 million yr of interaction with the massive planets, we find that a substantial fraction of test particles at low initial inclinations $i \lesssim 5^{\circ}$ have been promoted to orbits with higher inclinations, $i \sim 10^{\circ}$ (see Figs. 4 and 6). This promotion generally foretells an eventual close encounter with Neptune. Perhaps the peak at $i \approx 8^{\circ}$ in Figure $9 a$ represents KBOs recently delivered to $i \sim 0^{\circ}$ by some
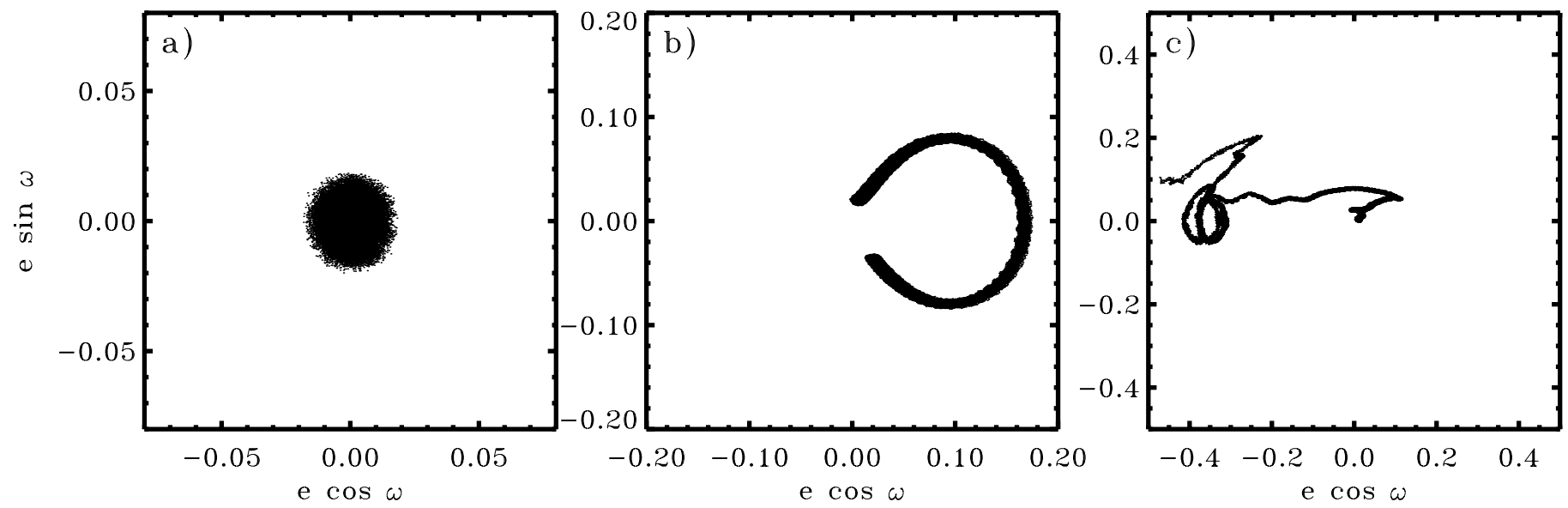

FIG. 8.-Pseudosurfaces of section for three particles with initial inclination $i=61^{\circ}$, semimajor axis $a=43$ AU, and eccentricity $e=0$, illustrating the three kinds of behavior we found near $i=61^{\circ}$. (a) Stable chaotic motion confined to low eccentricities. (b) Stable chaotic motion combined with slow libration around $\omega=90^{\circ}$ or $\omega=270^{\circ}$. (c) Particle which is ejected under the influence of the Kozai resonance. Note the three different scales. 

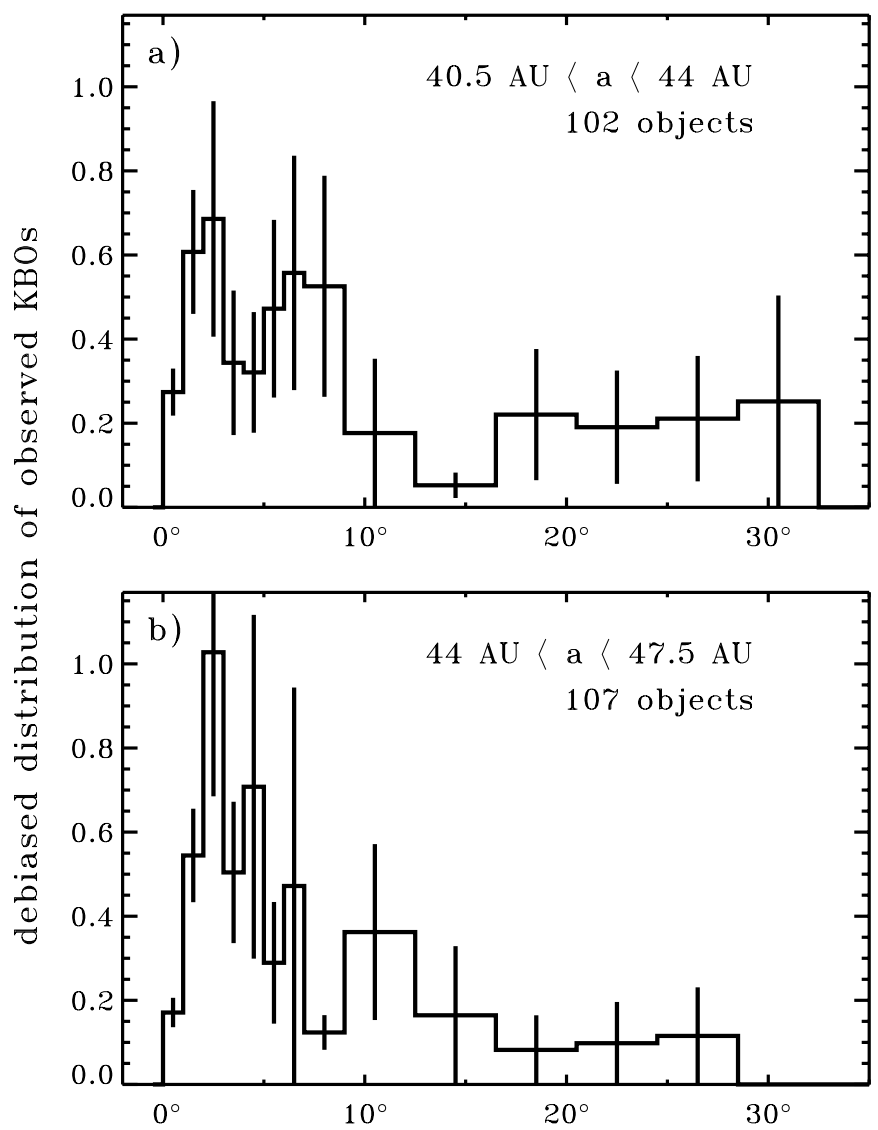

FIG. 9.-Debiased inclination distributions of the classical KBOs discovered at ecliptic latitude $<1^{\circ}$. (a) Objects with semimajor axis $40.5<$ $a<44$. (b) Objects with semimajor axis $44<a<47.5$. Distribution a reflects much more severe sculpting due to interaction with the massive planets.

self-interaction of the $\mathrm{KBO}$ disk and promoted to $i \approx 8^{\circ}$ on their way toward being hurled into Neptune's Hill sphere by the overlapped $\nu_{17}$ and $\nu_{18}$ secular resonances.

\section{COMET AND SCATTERED KBO FORMATION}

The classical Kuiper belt may be the primary source of short-period comets, Centaurs, and scattered Kuiper belt objects. Levison \& Duncan (1997) found that objects from the Kuiper belt that wander into the solar system after a close encounter with Neptune have a median dynamical lifetime of only $4.5 \times 10^{7} \mathrm{yr}$, so perhaps a substantial fraction of short-period comets and scattered KBOs were classical KBOs less than a billion years ago. To explore the origin of short-period comets, Centaurs, and scattered KBOs, we examined the particles in our integration that were ejected last from the classical Kuiper belt.

Figure 10 shows the initial semimajor axes, eccentricities, and inclinations of the particles in our integrations that encountered Neptune between $t=2$ billion and $t=4$ billion yr. Most $(85 \%)$ of this population of ejected objects originated on orbits with perihelion distances $q<39$ AU. Triangles show the initial orbits of particles ejected between $t=2$ billion and $t=3$ billion yr. Asterisks show the initial orbits of particles ejected between $t=3$ billion and $t=4$ billion yr.
We were surprised to find that the objects ejected from our simulation between $t=3$ billion yr and $t=4$ billion yr came exclusively from high initial inclinations $\left(i>15^{\circ}\right)$. Objects in our first batch encountered Neptune at a rate roughly proportional to $t^{-1}$ for the first 1 billion yr, in agreement with Holman \& Wisdom (1993) and Duncan et al. (1995). However, during the last billion years of the simulation, not one particle from low inclinations $\left(i<15^{\circ}\right)$ encountered Neptune, while 14 particles from higher inclinations $\left(15^{\circ}<i<33^{\circ}\right)$ encountered Neptune during this interval. We would expect $\sim 10$ encounters for the low-inclination group during that period if the rate remained proportional to $t^{-1}$.

Naturally, the overlapping secular resonances that create the large region of instability at low inclinations throughout the classical Kuiper belt affect the rate of Neptune encounters for low-inclination objects. These secular resonances remove low-inclination objects early on, so relatively few remain at $t>3$ billion yr to encounter Neptune; in the region $q<39$ AU almost twice as many high-inclination $\left(15^{\circ}<i<33^{\circ}\right)$ objects survive for 3 billion yr as low-inclination objects $\left(i<15^{\circ}\right)$. However, this selective removal of low-inclination objects does not explain why not a single low-inclination object in our simulation encountered Neptune from $t=3$ billion to $t=4$ billion yr. Enough lowinclination objects remain at $t=4$ billion yr to provide a significant flux of Neptune encounters.

But the secular resonances do not act alone. MMRs can protect the low-inclination objects from destruction at the hands of the overlapping secular resonances. The secular resonances may weed out all but the MMR-protected lowinclination objects in the first 3 billion yr, so that, after that time, the only available unprotected objects for them to eject have high inclinations.

Figure 3 shows that, at $a<44 \mathrm{AU}$, all but a few particles with $q<39$ AU that survive at low inclinations for 4 billion yr (Fig. 3) inhabit MMRs. High-inclination particles in this zone are spread more evenly; some high-inclination particles lie in MMRs, but many solid squares fall between the MMRs. Figure 2 shows that the real KBOs mimic the behavior of the test particles that survive 4 billion yr; 12 out of the 15 low-inclination $\left(i<15^{\circ}\right) \mathrm{KBOs}$ at $a<44 \mathrm{AU}$, $q<39$ AU with multiple-opposition orbits have observed semimajor axes and eccentricities within or near the numerically calculated boundaries of MMRs. The observed highinclination KBOs are spread more evenly in semimajor axis.

Our simulation suggests that today's short-period comets, Centaurs, and scattered KBOs originate in the highinclination KBO population. Therefore, short-period comets, Centaurs, and scattered KBOs should have a color distribution like the high-inclination KBOs when they leave the Kuiper belt. Trujillo \& Brown (2002) found that lowinclination $\mathrm{KBOs}$ are uniformly red $(B-R \approx 1.5-2.2$ for $\left.i<12^{\circ}\right)$, while high-inclination $\mathrm{KBOs}$ range from red to blue $\left(B-R \approx 1.0-2.0\right.$ for $\left.i>12^{\circ}\right)$.

Jewitt (2002) compared the colors of KBOs, Centaurs, comet nuclei, and candidate dead comets, and found that the Centaurs in his study had a wide range of colors. This range appears to be consistent with the color range of the high-inclination KBOs in (Trujillo \& Brown 2002), in agreement with our dynamical result. More color measurements of Centaurs may determine whether the color distribution of centaurs matches the color distribution of the high-inclination KBOs in detail. Jewitt's samples of comet nuclei and 


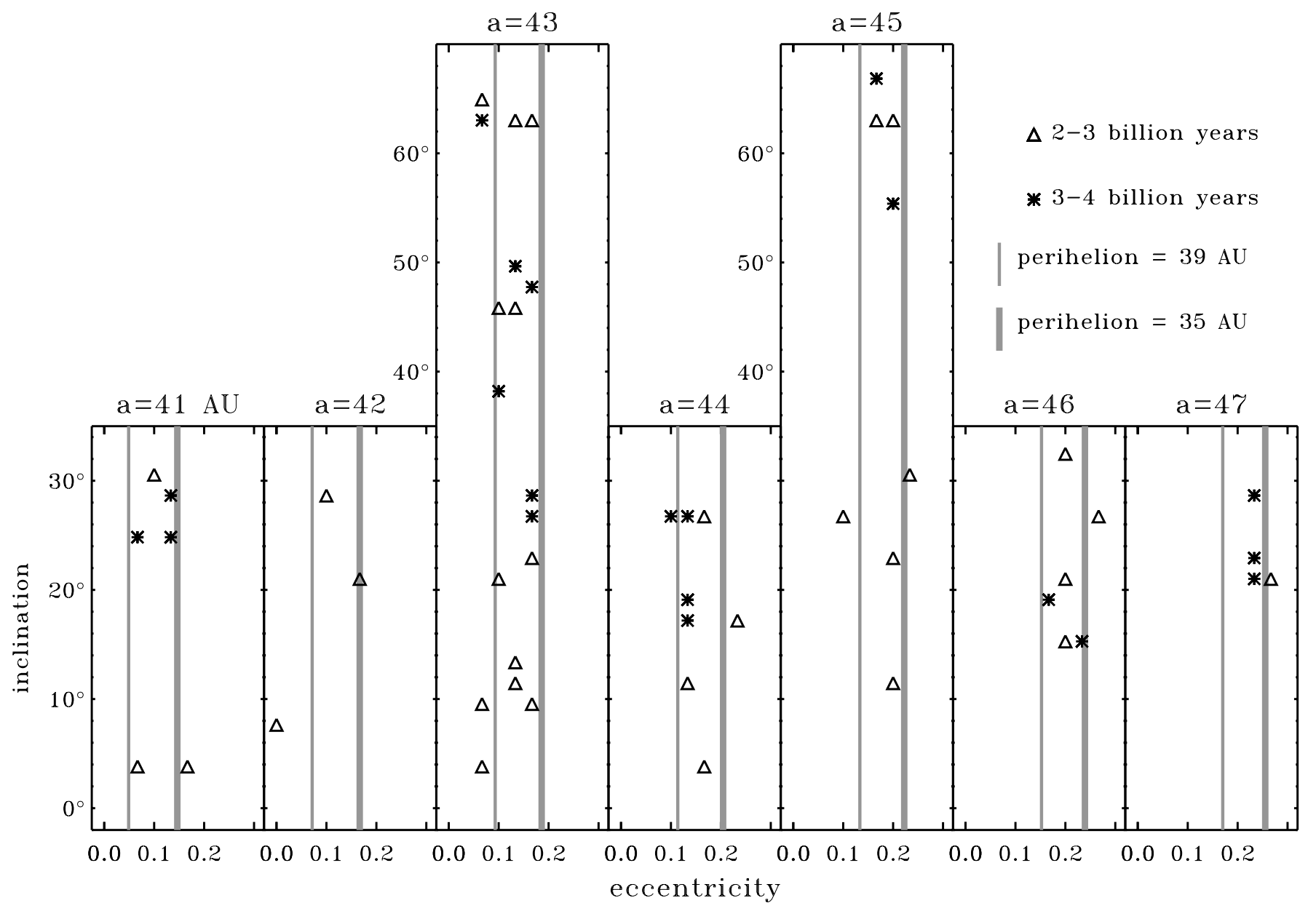

FIG. 10.- Initial orbits of particles that are removed late in the integrations when they enter Neptune's Hill sphere. Most of these particles originate on orbits with perihelia between 35 and $39 \mathrm{AU}$ (the vertical gray lines). Particles removed between $t=2$ billion and $t=3$ billion yr (triangles) come from all inclinations. Particles removed between $t=3$ billion yr and $t=4$ billion yr originated only at high inclinations $\left(i>15^{\circ}\right)$.

candidate dead comets had no objects as red as the red high-inclination KBOs; our study offers no solution to the problem of this missing ultrared matter.

\section{CONCLUSIONS}

Dynamical erosion is not the only long-term effect in the classical Kuiper belt; collisions may shape the distributions of KBO orbital elements (Stern \& Colwell 1997; Kenyon \& Luu 1998, 1999; Durda \& Stern 2000). But as the collision rate and the mass of the Kuiper belt decreased with time, the dynamical effects shown by our integrations must have begun to dominate the shaping of the classical Kuiper belt. We found that interactions with the massive planets preferentially deplete low-inclination objects in the inner half of the classical Kuiper belt, raising the mean inclination of the population of surviving objects. However, the outer half of the classical Kuiper belt $(a>44$ AU, $q<39$ AU) could have retained its ancient inclination distribution.

We also found that objects ejected from the classical Kuiper belt during the last 1 billion yr of our integration primarily come from initial orbits at high inclinations and eccentricities. This effect may be caused indirectly by $\nu_{17}$ and $\nu_{18}$, which remove many low-inclination particles at early times and weed out those unprotected by MMRs. This finding implies that Centaurs, short-period comets, and scattered KBOs should have an initial color distribution like that of the high-inclination KBOs. Measuring of the colors of more Centaurs could test this hypothesis.

Perhaps the high-inclination KBOs separated from the primordial KBOs objects during a special event early in the lifetime of the solar system. If today's active comets arise mainly from high-inclination KBOs, we have this event to thank for their inner solar system company.

Thanks to Edo Berger, John-Philippe Berger, John Cartwright, Roy Gal, Richard Ellis, Brian Jacoby, Ken Jucks, David Kaplan, Robert Kirshner, David Latham, Lori Lubin, Brian Mason, Mark Metzger, Rafael Milan-Gabet, Irene Porro, Nick Scoville, Patrick Shopbell, John Sievers, Wesley Traub, Pat Udomprasert, and Bill Wyatt for donating CPU time for this project. Thanks to Hal Levison, Scott Kenyon, and Ed Thommes for helpful discussions. This work was performed in part under contract with the Jet Propulsion Laboratory through the Michelson Fellowship program funded by NASA as an element of the Planet Finder Program. JPL is managed for NASA by the California Institute of Technology. 
Brown, M. E. 2001, AJ, 121, 2804

Cohen, C. J., Hubbard, E. C., \& Oesterwinter, C. 1973, Elements of the Outer Planets for One Million Years (Washington: GPO)

Duncan, M. J., Levison, H. F., \& Budd, S. M. 1995, AJ, 110, 3073

Duncan, M., Quinn, T., \& Tremaine, S. 1988, ApJ, 328, L69

Durda, D. D., \& Stern, S. A. 2000, Icarus, 145, 220

Fernández, J., A. 1980, MNRAS, 192, 481

Ferraz-Mello, S., \& Sato, M. 1989, A\&A, 225, 541

Gladman, B., \& Duncan, M. J. 1990, AJ, 100, 1696

Gladman, B., Kavelaars, J. J., Petit, J.-M., Morbidelli, A., Holman, M. J., \& Loredo, T. 2001, AJ, 122, 1051

Henon, M. 1983, in Chaotic Behavior of Deterministic Systems, ed. G. Iooss, R. H. G. Helleman, \& R. Stora (Amsterdam: North-Holland), 53

Holman, M. \& Wisdom, J. 1993, AJ, 105, 1987

Ida, S., Larwood, J., \& Burkert, A. 2000, ApJ, 528, 351

Jewitt, D. 2002, AJ, 123, 1039

Jewitt, D., \& Luu, J. 1995, AJ, 109, 1867

Jewitt, D., Luu, J., \& Chen, J. 1996, AJ, 112, 1225

Jewitt, D., Luu, J., \& Trujillo, C. 1998, AJ, 115, 2125

Kenyon, S. J. 2002, PASP, 114, 265

Kenyon, S. J., \& Luu, J. X. 1998, AJ, 115, 2136

\section{REFERENCES}

Kenyon, S. J., \& Luu, J. X. 1999, AJ, 118, 1101

Knezevic, Z., Milani, A., Farinella, P., Froeschle, C., \& Froeschle, C. 1991, Icarus, 93, 316

Kozai, Y. 1962, AJ, 67, 591

Levison, H., \& Duncan, M. J. 1993, ApJ, 406, L35 1994, Icarus, 108,18 1997, Icarus, 127, 13

Malhotra, R. 1995, AJ, 110, 420 1996, AJ, 111, 504

Morbidelli, A. 1997, Icarus, 127, 1

Morbidelli, A., Thomas, F., \& Moons, M. 1995, Icarus, 118, 322

Nagasawa, M., \& Ida, S. 2000, AJ, 120, 3311

Petit, J.-M., Morbidelli, A., \& Valsecchi, G. B. 1999, Icarus, 141, 367

Stern, S. A., \& Colwell, J. E. 1997, ApJ, 490, 879

Thomas, F., \& Morbidelli, A. 1996, Celest. Mech. Dyn. Astron., 64, 209

Thommes, E. W., Duncan, M. J., \& Levison, H. F. 2002, AJ, 123, 2862

Torbett, M. 1989, AJ, 98, 1477

Torbett, M., \& Smoluchowski, R. 1990, Nature, 345, 49

Trujillo, C., \& Brown, M. E. 2001, ApJ, 554, L95 2002, ApJ, 566, L125

Trujillo, C. A., Jewitt, D. C., \& Luu, J. X. 2001, AJ, 122, 457

Wisdom, J., \& Holman, M. 1991, AJ, 102, 1528 